\title{
DC-Bias Allocation in Cooperative VLC Networks via Joint Information and Energy Transfer
}

\author{
Mohanad Obeed*, Hayssam Dahrouj», Anas M. Salhab*, Salam A. Zummo*, and Mohamed-Slim Alouini ${ }^{+}$ \\ *King Fahd University of Petroleum \& Minerals, Dhahran, Eastern Province, Saudi Arabia \\ Effat University, Jeddah, Makkah Province, Saudi Arabia \\ ${ }^{+}$King Abdullah University of Science and Technology, Thuwal, Makkah Province, Saudi Arabia \\ Email: * $\{$ g201106250, salhab, zummo $\} @$ kfupm.edu.sa ${ }^{\star}$ hayssam.dahrouj@gmail.com, ${ }^{+}$slim.alouini@kaust.edu.sa
}

\begin{abstract}
In order to meet the new escalating demand for high data rate services and applications, visible light communication (VLC) has emerged as a promising solution for the fifth-generation (5G) wireless networks and beyond. Consider a VLC network, where multiple access points (APs) serve both energy-harvesting users (EHUs), i.e., users which harvest energy from light intensity, and information-users (IUs), i.e., users which gather data information. The performance of the system becomes a function of the direct current (DC) bias values allocated to each AP. After adopting a zero-forcing (ZF) precoding approach to cancel the inter-cell interference, the paper formulates the problem of maximizing the network harvested energy subject to individual harvested energy and data rate constraints at the EHUs and IUs, respectively, so as to determine the DC bias of every AP. The paper then proposes solving such a difficult non-convex optimization problem using an iterative approach. The proposed algorithm uses well-chosen approximations of the objective and constraints functions, and compensates for the approximations using proper outer-loop updates. The paper further proposes a sub-optimal heuristic which provides a feasible, yet simple, solution to the problem. Numerical results illustrate the convergence of our proposed algorithms, and highlight the significant performance improvement of the proposed algorithm as compared to the proposed baseline approach.
\end{abstract}

Index Terms - Visible light communication, energy harvesting, convex optimization.

\section{INTRODUCTION}

Much attention has recently been paid to energy-harvesting techniques at user-equipment devices, either from exploiting the surrounding environment, or by transferring wireless power. Energy harvesting is the capability of converting the radio frequency $(\mathrm{RF})$ signals or light intensity into electrical voltage/current. With the advent of the era of the Internetof-things (IoT), the demand for transferring the power and enabling IoT devices to harvest energy using light or RF transmission is increasing, especially in indoor applications where smart buildings, health monitoring, and sensors devices applications become abundant.

Concurrently, the growing demand for high data rate and licence-free spectrum applications has pushed researchers to investigate visible light communication (VLC) techniques as a supplementary technology for indoor communication. This is especially the case because of the scarcity of the available RF spectrum due to ultra-dense network deployment. VLC, further, provides higher data rate, larger energy efficiency, lower battery consumption, and smaller latency as compared to RF-based networks [1]. VLC can be, indeed, safely used in sensitive environments such as chemical plants, aircraft, and hospitals [2]. Despite the small coverage of the transmitters in VLC systems, an exhaustive reuse of frequency can be implemented, with a relatively small effect on the co-channel interference [3], [4]. This paper evaluates the benefit of a particular VLC-based scheme that addresses the energy harvesting problem under a setup which considers the coexistence of both IUs and EHUs, and addresses the problem of maximizing the harvested energy by means of properly adjusting the DC bias values at the transmitters.

Investigating the VLC system efficiency has been a timely topic of interest of late [5]-[11]. Reference [5] verifies experimentally the light energy harvested at the mobile phone, when equipped with a commercial solar panel in indoor environments. Authors in [5] show that the devices that are directly exposed to the indoor light can be charged to a satisfied level. In [6] and [7], a dual-hop hybrid VLC/RF communication system is studied as a means to reach out to the out-of-coverage user. References [6], [7] show that visible light can be used in the first hop to transfer both data information and energy to the relay. The relay, then, forwards the data to the destination using the resulting harvested energy. References [8], [9] maximize the sum-rate utility of a VLC system consisting of one AP and $K$ users, subject to individual QoS constraints. Reference [8] assumes that user $k$ receives the information in their assigned time slot, and receives the power within the time slots that are assigned for other users. Reference [9], on the other hand, proposes solving the problem by allocating the optical intensity and time slots through adopting a looser upper bound on the individual required harvested energy. The authors in [10] characterize the outage performance of a hybrid VLC-RF system, where the visible light is used for the downlink to transfer the energy and data to the users, and then the users use the harvested energy to transmit a RF signal in the uplink.

All the above papers use the alternating current (AC) component for harvesting the energy at users, where the DC component of the transmitted light is fixed and readily used to harvest energy. Toward this direction, reference [11] considers a network that enables simultaneous light-wave information and power transfer (SLIPT) and maximizes the harvested energy under QoS constraints, so as to determine the DC in a portion of time, given that DC bias in the remain- 


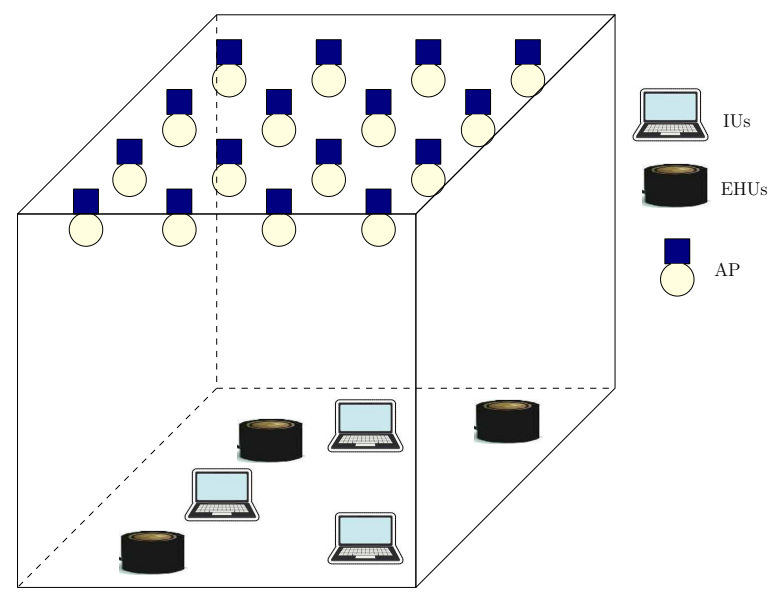

Fig. 1. System model (an example of user distribution when $N_{u, 1}=3$, $N_{u, 2}=3$, and $N_{A}=16$ ).

ing time is fixed for the purpose of transferring energy only. Reference [11], however, is restricted to a single transmitter and a single receiver only. The authors in [11], further, do not impose any energy constraints on users operation.

Unlike all aforementioned papers, our paper investigates the DC bias allocation for simultaneous power and data transfer in multiple APs multi-user VLC systems. Consider a VLC network, where multiple access points serve both EHUs (e.g. sensors or IoT devices), and IUs (e.g. laptops, mobile phones, etc.), so as to best capture the multi-diverse applications schemes expected in $5 \mathrm{G}$ systems. For simplicity, the paper adopts the zero-forcing (ZF) precoding approach to cancel intra-cell interference; see [12], [13] and references therein. The paper then addresses the problem of maximizing the network harvested energy subject to individual harvested energy and data rate constraints at the EHUs and IUs, respectively, so as to determine the DC bias of every AP. The paper proposes solving such a difficult non-convex optimization problem using an iterative approach, which uses well-chosen approximations of the objective and constraints functions, and then compensates for the approximations using proper outerloop updates. The paper, further, presents a suboptimal, yet simple, solution to the problem. Simulations results highlight the convergence of our proposed algorithms, and suggest that an appreciable harvested energy can be reached by optimizing the DC bias in VLC schemes.

\section{System AND Channel Models}

\section{A. System Model}

Consider an indoor VLC system consisting of $N_{A}$ VLC access points (APs), which serve $N_{u}$ users in total. Among the $N_{u}$ users, $N_{u, 1}$ users are IUs, and $N_{u, 2}$ are EHUs, i.e., $N_{u}=N_{u, 1}+N_{u, 2}$. The paper considers the case where $N_{u, 1}<N_{A}$, and adopts a zero-forcing (ZF) approach to cancel the intra-cell interference, so as to simplify the mathematical tractability of the problem. Let $\mathbf{s} \in \Re^{N_{u, 1} \times 1}$ be the vector hosting the information of the $N_{u, 1}$ users, and let $\mathbf{G} \in \Re^{N_{A} \times N_{u, 1}}$ be the precoding matrix associated with s. In other terms, the electrical signal transmitted from AP $i$ can be written as $x_{i}=\mathbf{G}(i,:) \overline{\mathbf{P}} \mathbf{s}$, where $\mathbf{G}(i,:)$ is the $i^{t h}$ row of the matrix $\mathbf{G}$ and $\overline{\mathbf{P}}=\operatorname{diag}\left(\left[\sqrt{P_{1}}, \sqrt{P_{2}}, . ., \sqrt{P_{N_{u, 1}}}\right]\right)$, where $P_{j}$ is the electronic power assigned to the $j$ th user's message. Denote by $b_{i}$ the DC bias, which must be added to $x_{i}$ to avoid the resulting non-positive signals [11]. The electrical signal, afterwards, modulates the optical intensity of the light-emitting diodes (LEDs) at AP $i$. The transmitted signal at AP $i$ can, therefore, be written as:

$$
y_{t, i}=P_{\text {opt }}\left(b_{i}+x_{i}\right),
$$

where $P_{\text {opt }}$ is the LEDs power at each AP. Let $I_{L}$ and $I_{H}$ be the minimum and the maximum input bias currents, respectively, i.e., $b_{i} \in\left[I_{L}, I_{H}\right]$. In a VLC network, the LED has to operate in the linear region so that the optical power at its output is a linear function of the input voltage. Thus, the peak amplitude of the modulated signal $x_{i}$, denoted by $A_{i}$, must satisfy the following constraint:

$$
A_{i} \leq \min \left(b_{i}-I_{L}, I_{H}-b_{i}\right) .
$$

Constraint (2) implies that $A_{i}$ must satisfy two constraints, which are $A_{i}+b_{i} \leq I_{H}$ and $b_{i}-A_{i} \geq I_{L}$, to guarantee that the input electrical signal to the LED must be within the range of the linear region LED operation.

\section{B. Channel Model}

The paper adopts a line-of-sight (LoS) VLC channel model as in [14]. More precisely, the channel between the $i^{\text {th }}$ LED and the $j^{t h}$ user, denoted by $h_{i, j}^{(v)}$, can be expressed as follows:

$$
h_{i, j}^{(v)}=\frac{(m+1) A_{p}}{2 \pi d_{i, j}^{2}} \cos ^{m}(\phi) g_{o f} f(\theta) \cos (\theta),
$$

where $m$ is the Lambertian index that is given by $m=$ $-1 / \log _{2}\left(\cos \left(\theta_{1 / 2}\right)\right.$, where $\theta_{1 / 2}$ is the half intensity radiation angle, $A_{p}$ is the physical area of the PD, $d_{i, j}$ is the distance between the $i^{t h}$ LED and the $j^{t h}$ user, $g_{o f}$ is the gain of the optical filter, $\phi$ is the angle of radiance at the LED, $\theta$ is the angle of incidence at the photo-detector (PD), and where $f(\theta)$ is the optical concentrator gain, which is a function of $\theta$ that is given by:

$$
f(\theta)= \begin{cases}\frac{n^{2}}{\sin ^{2}(\Theta)}, & \theta \leq \Theta \\ 0, & \theta>\Theta,\end{cases}
$$

where $n$ is the refractive index and $\Theta$ is the semi-angle of the field-of-view (FoV) of the PD. The assumption of the existence of LoS is aligned with the majority of the existing works on VLC-based systems. In fact, such assumption does not affect the algorithm proposed in the next section, as the channel is rather used in a more generic form. For the sake of completeness, one can simply set $h_{i, j}^{(v)}$ to (3) when the LoS path is available, and to zero otherwise.

After removing the DC bias at the receiver side, the received signal vector at the users from all APs is given by:

$$
\mathbf{Y}_{r}=\rho \mathbf{H G} \overline{\mathbf{P}} \mathbf{s}+\mathbf{n}
$$


where $\rho$ is the optical-to-electric conversion factor, $\mathbf{H} \in$ $\mathbb{R}^{N_{u} \times N_{A}}$ is the channel attenuation matrix that is assumed to be known at APs, $\mathbf{G}$ is the pre-coding matrix used to diagonalize the channel matrix, i.e., $\mathbf{G}=\mathbf{H}^{T}\left(\mathbf{H} \mathbf{H}^{T}\right)^{-1}$, and $\mathbf{n}$ is the noise vector which includes both the thermal noise and the shot noise at the user, which can be modelled as zeromean complex-valued AWGN with variance $\sigma^{2}=N_{0} W$, where $W$ is the modulation bandwidth, and $N_{0}$ is the noise power spectral density.

A tight lower bound on the network sum-rate at the $N_{u, 1}$ IUs can then be written as [15]:

$$
\eta=\beta \sum_{j=1}^{N_{u, 1}} \log \left(1+\frac{e\left(\rho^{2}\right) P_{j}}{2 \pi W N_{0}}\right),
$$

where $\beta=W / 2$ is a constant and $e$ is the constant exponential (Euler's number). It is important to note that, from Equation (5), we can define the relation between the transmit power at AP $i$ and the assigned powers of the messages as:

$$
p_{i}=\sum_{j=1}^{N_{u, 1}} g_{i, j}^{2} P_{j}
$$

where $g_{i, j}$ is the $(i, j)$-th element of matrix G. Based on equation (1), the transmit power at the $i$ th AP is further related to the peak amplitude of the modulated signal as follows:

$$
p_{i}=\left(P_{o p t} A_{i}\right)^{2}
$$

\section{Energy Harvesting Signals}

For the EHUs, the DC component of the received signal is blocked by a capacitor and forwarded to the energy harvesting circuit [11]. The harvested energy is given by [5]:

$$
E=f I_{D C} V_{o c}
$$

where $f$ is the fill factor (typically around 0.75 ), and $I_{D C}$ is the received DC current which is given at the $j$ th user by:

$$
I_{D C}=\rho P_{o p t} \mathbf{h}_{j}^{T} \mathbf{b},
$$

where $\mathbf{b}=\left[b_{1}, b_{2}, \ldots, b_{N_{A}}\right]$ is the DC bias vector at APs, $\mathbf{h}_{j}$ is the channel vector from all APs to the user $j$, and

$$
V_{o c}=V_{t} \ln \left(1+\frac{I_{D C}}{I_{0}}\right)
$$

where $V_{t}$ is the thermal voltage, and $I_{0}$ is the dark saturation current of the PD. Hence, the harvested energy at user $j$ is given by:

$$
E_{j}=f \rho P_{o p t} V_{t} \mathbf{h}_{j}^{T} \mathbf{b} \ln \left(1+\frac{\rho \mathbf{h}_{j}^{T} P_{o p t} \mathbf{b}}{I_{0}}\right) .
$$

It is important to note that the DC bias $b_{i}$ at the $i$ th AP must be greater than or equal to $\frac{I_{H}+I_{L}}{2}$. This is because decreasing $b_{i}$ to be less than $\frac{I_{H}+I_{L}}{2}$ results in decreasing the harvested energy (12). It also decreases $A_{i}$ (based on (2)), which decreases the transmit power $p_{i}$ that leads to a decrease in the sum-rate (6). $b_{i}$, therefore, should satisfy: $b_{i} \geq$
$\frac{I_{H}+I_{L}}{2}$, and $\min \left(I_{H}-b_{i}, b_{i}-I_{L}\right)$ becomes equal to $I_{H}-b_{i}$. Therefore, the relation (2) between $A_{i}$ and $b_{i}$ becomes:

$$
A_{i} \leq I_{H}-b_{i}
$$

In addition, if the optimal $b_{i}$ satisfies $b_{i} \leq I_{H}-A_{i}$, it can be increased to have $b_{i}=I_{H}-A_{i}$, which increases the objective function without violating the QoS constraints. Hence, the inequality in (13) is satisfied with equality:

$$
A_{i}=I_{H}-b_{i}
$$

\section{PROBLEM ForMULATION}

The optimization problem considered in this paper consists of maximizing the total harvested energy at all EHUs, subject to individual harvested energy and data rate constraints at the EHUs and IUs, respectively, by means of determining the optimal DC bias vector $\mathbf{b}$ at the APs. From Equations (12) and (14), it can be readily seen that while increasing the DC biases results in increasing the total harvested energy at the EHUs, it decreases the data rate at the IUs (because the transmit power for the information signal at AP $i$ is given by $\left.p_{i}=\left(P_{o p t} A_{i}\right)^{2}\right)$. Hence, our problem of interest can be thought of as an initiative to achieve a relative trade-off between the total harvested energy and the achievable data rates at IUs. In addition, equations (8) and (14) illustrate a one-to-one mapping between the DC bias and the power allocation, which shows the problem this paper considers jointly finds the the transmit power and the corresponding DC bias at each AP. Mathematically, the considered optimization problem can be formulated as follows:

$$
\begin{array}{ll}
\max _{\mathbf{b}} & \sum_{j=1}^{N_{u, 2}} f \rho P_{o p t} V_{t} \mathbf{h}_{j}^{T} \mathbf{b} \ln \left(1+\frac{\rho P_{o p t} \mathbf{h}_{j}^{T} \mathbf{b}}{I_{0}}\right) \\
\text { s.t. } & \beta \log \left(1+\frac{e\left(\rho^{2}\right) P_{j}}{2 \pi W N_{0}}\right) \geq R_{t h, j}, \\
& j=1, \ldots, N_{u, 1} \\
& f \rho P_{o p t} V_{t} \mathbf{h}_{j}^{T} \mathbf{b} \ln \left(1+\frac{\rho P_{o p t} \mathbf{h}_{j}^{T} \mathbf{b}}{I_{0}}\right) \geq E_{t h, j}, \\
& j=1, \ldots, N_{u, 2} \\
& p_{i}=P_{o p t}^{2}\left(I_{H}-b_{i}\right)^{2}, \quad i=1, . ., N_{A}, \\
& \frac{I_{H}+I_{L}}{2} \leq b_{i} \leq I_{H}, \quad i=1, . ., N_{A},
\end{array}
$$

where the optimization is over the DC bias vector $\mathbf{b}$, where $R_{t h, j}$ in (15b) is the minimum required data rate at the $j$ th user, and where $E_{t h, j}$ in $(15 \mathrm{c})$ is the minimum required energy to be harvested by user $j$.

The above optimization problem is a difficult non-convex optimization problem as it maximizes a non-concave utility function. The paper next proposes solving the problem by reformulating and solving it using an iterative approach.

\section{Algorithms}

This sections presents a numerical solution to solve problem (15). The solution hinges upon reformulating the problem as a function of the DC bias only, after plugging the expression of $p_{i}$ (as given in (15d)) in the rest of the constraints. 
The paper then proposes a heuristic, yet efficient, algorithm of the reformulated problem by first solving an approximated problem, and correcting for the approximation in an outer loop update. For the sake of comparison, the paper further proposes a simple baseline approach, which guarantees a feasible solution to (15). Simulations results illustrate the convergence of the algorithms, and highlight the performance improvement of the proposed algorithm as compared to the proposed baseline approach.

\section{A. Problem Reformulation}

First, to simplify the considered optimization problem, we should express the transmit powers $p_{i}, i=1, \ldots, N_{A}$ and the messages' powers $P_{j}, j=1, \ldots, N_{u, 1}$ in terms of the DC-bias vector. Based on Equation (7), constraints in (15d) can be rewritten as $\sum_{j=1}^{N_{u, 1}} g_{i, j}^{2} P_{j}=P_{o p t}^{2}\left(I_{H}-b_{i}\right)^{2}, \quad i=$ $1, \ldots, N_{A}$. Define matrix $\hat{\mathbf{G}}=\left[\hat{\mathbf{g}}_{1}^{T} ; \hat{\mathbf{g}}_{2}^{T} ; \ldots ; \hat{\mathbf{g}}_{N_{A}}^{T}\right]$, where $\hat{\mathbf{g}}_{i}^{T}=$ $\left[g_{i, 1}^{2}, g_{i, 2}^{2}, \ldots, g_{i, N_{u, 1}}^{2}\right]$. Hence, constraints in $(15 \mathrm{~d})$ can be expressed in one constraint, which is $\hat{\mathbf{G P}}=P_{o p t}^{2}\left(I_{H}^{2} \mathbf{1}_{N_{A}}-\right.$ $\left.2 I_{H} \mathbf{b}+\mathbf{b} \odot \mathbf{b}\right)$, where $\mathbf{P}$ is the messages' power vector, where $\mathbf{1}_{N_{A}}$ is the one vector with length $N_{A}$, and where the operator $\odot$ denotes the vector component-wise multiplication. Let $\mathbf{F}=$ $\left(\hat{\mathbf{G}}^{T} \hat{\mathbf{G}}\right)^{-1} \hat{\mathbf{G}}^{T}$. The $j$ th constraint can then be expressed as follows:

$$
P_{j}=P_{o p t}^{2}\left(\mathbf{f}_{j}^{T} \mathbf{1}_{N_{A}} I_{H}^{2}-2 I_{H} \mathbf{f}_{j}^{T} \mathbf{b}+\mathbf{b}^{T} \overline{\mathbf{F}}_{j} \mathbf{b}\right)
$$

where $\mathbf{f}_{j}^{T}$ is the $j$ th row of the matrix $\mathbf{F}$ and $\overline{\mathbf{F}}_{j}=\operatorname{diag}\left(\mathbf{f}_{j}^{T}\right)$. Using the relation in (16), the $j$ th Constraint in (15b) can be written, in terms of the DC-bias vector, as:

$$
\mathbf{f}_{j}^{T} \mathbf{1}_{N_{A}} I_{H}^{2}-2 I_{H} \mathbf{f}_{j}^{T} \mathbf{b}+\mathbf{b}^{T} \overline{\mathbf{F}}_{j}^{T} \mathbf{b} \geq P_{j, \min }
$$

where $P_{j, \min }=\frac{\left(2^{\frac{R_{t h, j}}{\beta}}-1\right) 2 \pi W N_{0}}{P_{o p t}^{2} e \rho^{2}}$. Problem (15) becomes then equivalent to the following optimization problem:

$$
\begin{aligned}
& \max _{\mathbf{b}} \sum_{j=1}^{N_{u, 2}} f \rho P_{o p t} V_{t} \mathbf{h}_{j}^{T} \mathbf{b} \ln \left(1+\frac{\rho P_{o p t} \mathbf{h}_{j}^{T} \mathbf{b}}{I_{0}}\right) \\
& \text { s.t. } \mathbf{b}^{T} \overline{\mathbf{F}}_{j} \mathbf{b} \geq 2 I_{H} \mathbf{f}_{j}^{T} \mathbf{b}+P_{j, m i n}-\mathbf{f}_{j}^{T} \mathbf{1}_{N_{A}} I_{H}^{2} \\
& \quad j=1, . ., N_{u, 1} \\
& f \rho P_{o p t} V_{t} \mathbf{h}_{j}^{T} \mathbf{b} \ln \left(1+\frac{\rho P_{o p t} \mathbf{h}_{j}^{T} \mathbf{b}}{I_{0}}\right) \geq E_{t h, j} \\
& \quad j=1, . ., N_{u, 2} \\
& \frac{I_{H}+I_{L}}{2} \leq b_{i} \leq I_{H}, \quad i=1, . ., N_{A} .
\end{aligned}
$$

Problem (18) is still a non-convex optimization problem. Hence, we next propose a novel method that solves problem (18) by approximating it to a convex problem, and then compensate for that approximation in the outer loop.

\section{B. Approximation}

The idea here is to estimate the initial values for the DC bias vector, then replace some terms in the non-concave functions by the estimated vector, so as to convexify problem (18). Specifically, for the objective function and constraints in (18c), replace the log expressions by their estimated values, which converts them to linear functions. Define $\hat{b}_{i}=\frac{I_{H}+I_{L}}{2}$, i.e., let $\hat{\mathbf{b}}=\frac{I_{H}+I_{L}}{2} \mathbf{1}_{N_{A}}$ be the estimated DC bias vector. In problem (15), replace the log terms by the fixed term $z_{j}(\hat{\mathbf{b}})=\ln \left(1+\frac{\rho P_{o p t} \mathbf{h}_{j}^{T} \hat{\mathbf{b}}}{I_{0}}\right)$, which is now given. Hence, problem (15) can be approximated as follows:

$$
\begin{array}{ll}
\max _{\mathbf{b}} & \sum_{j=1}^{N_{u, 2}} z_{j}(\hat{\mathbf{b}}) f \rho P_{o p t} V_{t} \mathbf{h}_{j}^{T} \mathbf{b} \\
\text { s.t. } & \mathbf{b}^{T} \overline{\mathbf{F}}_{j} \mathbf{b} \geq 2 I_{H} \mathbf{f}_{j}^{T} \mathbf{b}+P_{j, \min }-\mathbf{f}_{j}^{T} \mathbf{1}_{N_{A}} I_{H}^{2}, \\
& j=1, . ., N_{u, 1} \\
& z_{j}(\hat{\mathbf{b}}) f \rho P_{o p t} V_{t} \mathbf{h}_{j}^{T} \mathbf{b} \geq E_{t h, j} \\
& j=1, . ., N_{u, 2} \\
& \frac{I_{H}+I_{L}}{2} \leq b_{i} \leq I_{H}, \quad i=1, . ., N_{A} .
\end{array}
$$

The objective function and constraints in (19c) are linear functions. By further approximating constraints (19b) to be

$$
\hat{\mathbf{b}}^{T} \overline{\mathbf{F}}_{j} \mathbf{b} \geq 2 I_{H} \mathbf{f}_{j}^{T} \mathbf{b}+P_{j, \min }-\mathbf{f}_{j}^{T} \mathbf{1}_{N_{A}} I_{H}^{2}, \quad j=1, . ., N_{u, 1},
$$

the optimization problem (19) can be rewritten as:

$$
\begin{array}{ll}
\max _{\mathbf{b}} & \sum_{j=1}^{N_{u, 2}} z_{j}(\hat{\mathbf{b}}) f \rho P_{\text {opt }} V_{t} \mathbf{h}_{j}^{T} \mathbf{b} \\
\text { s.t } \quad & \hat{\mathbf{b}}^{T} \overline{\mathbf{F}}_{j} \mathbf{b} \geq 2 I_{H} \mathbf{f}_{j}^{T} \mathbf{b}+P_{j, \min }-\mathbf{f}_{j}^{T} \mathbf{1}_{N_{A}} I_{H}^{2}, \\
& j=1, \ldots, N_{u, 1} \\
& z_{j}(\hat{\mathbf{b}}) f \rho P_{o p t} V_{t} \mathbf{h}_{j}^{T} \mathbf{b} \geq E_{t h, j} \\
& j=1, \ldots, N_{u, 2} \\
& \frac{I_{H}}{2} \leq b_{i} \leq I_{H}, \quad i=1, \ldots, N_{A} .
\end{array}
$$

\section{Iterative Algorithm}

It can be seen that problem (20) is a linear programming (LP) problem, which can be efficiently solved using existing solvers [16]. To mitigate the effect of the approximation on the original optimization problem, we propose in the following an iterative algorithm that aims to compensate for the proposed approximations, and enhance the algorithmic performance as the number of iterations increases.

\footnotetext{
Algorithm 1 Find the vector $\mathbf{b}$

1) Find the estimated DC bias vector by putting $\hat{\mathbf{b}}=$ $\frac{I_{H}+I_{L}}{2} \mathbf{1}_{N_{A}}$.

2) Solve Problem (20), with the given $\hat{\mathbf{b}}$, and find the solution $\mathbf{b}$.

3) if $\|\hat{\mathbf{b}}-\mathbf{b}\|^{2}>\epsilon$ or the maximum iteration is not reached, update $\hat{\mathbf{b}}=\mathbf{b}$ and go to Step 2 .
}

The main idea of Algorithm 1 is to update the estimated DC bias vector in each iteration by equating it with the resulted DC bias vector from the previous iteration. This process continues until the norm of the difference between the estimated and the resulted DC bias vectors becomes less than a nominal threshold $\epsilon$. Since the proposed Algorithm 1 solves a series of LPs at each iteration, several feasible algorithms 
TABLE I

Simulation PARAMETERS

\begin{tabular}{lc}
\hline Name of the Parameter & Value of the Parameter \\
\hline & \\
Maximum bandwidth of VLC AP, $B$ & $20 \mathrm{MHz}$ \\
The physical area of a PD for IUs, $A_{p}$ & $0.1 \mathrm{~cm}^{2}$ \\
The physical area of a PD for EHUs, $A_{p}$ & $0.04 \mathrm{~m}^{2}$ \\
Half-intensity radiation angle, $\theta_{1 / 2}$ & $60^{\circ}$ \\
FoV semi-angle of PD, $\Theta$ & $45^{\circ}-65^{\circ}$ \\
Gain of optical filter, $g_{o f}$ & 1 \\
Refractive index, $n$ & 1.5 \\
Optical-to-electric conversion factor, $\rho$ & $0.53[\mathrm{~A} / \mathrm{W}]$ \\
Maximum input bias current, $I_{H}$ & $12 \mathrm{~mA}$ \\
Minimum input bias current, $I_{L}$ & $0 \mathrm{~A}$ \\
Fill factor, $f$ & 0.75 \\
LEDs' power, $P_{o p t}$ & $10 \mathrm{~W} / \mathrm{A}$ \\
Thermal voltage, $V_{t}$ & $25 \mathrm{mV}$ \\
Dark saturation current of the PD, $I_{0}$ & $10^{-10} \mathrm{~A}$ \\
Noise power spectral density of LiFi, $N_{0}$ & $10^{-22} \mathrm{~A} / \mathrm{Hz}$ \\
Room size & $8 \times 8$ \\
Room height & $3 \mathrm{~m}$ \\
User height & 0.9 \\
Number of APs & $4 \times 4$ \\
Number of users & $6-20$ \\
\hline
\end{tabular}

can be used to solve the problem with low computational complexity [17]. Simulations results further illustrate the fast convergence speed of the proposed algorithm.

\section{Baseline Algorithm}

As a benchmark baseline, we now propose a simple, yet feasible, solution to problem (15). In this approach, for simplicity, the DC-bias values at APs are assumed to be equal. Based on this assumption and within the bounds of the DC-bias values, we propose to search for the suitable DCbias value that achieves the QoS constraints and, at the same time, maximizes the harvested energy. We adopt the bisection algorithm to optimize the DC-bias value that must be within the range $\frac{I_{H}+I_{L}}{2} \leq b \leq \frac{I_{H}}{2}$. As discussed earlier, increasing the DC-bias at the APs leads to increasing the harvested energy at the EHUs, and also decreasing the information rates at the IUs. Therefore, the idea of this algorithm is to find the DC-bias range that achieves the tradeoff between constraints. Then within this range, we find the DC-bias that maximizes the total harvested energy. Such process can be summarized by Algorithm 2 steps below.

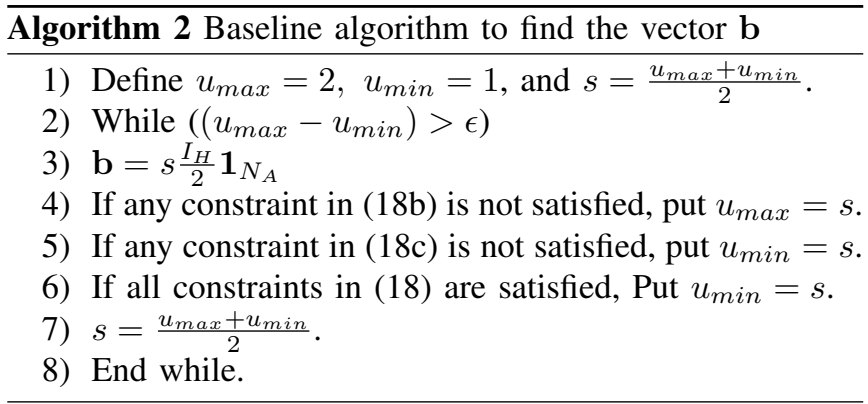

\section{Simulation Results}

This section evaluates the performance and convergence behavior of the proposed algorithm by illustrating how the number of users, the FoV, and the LEDs' power affect

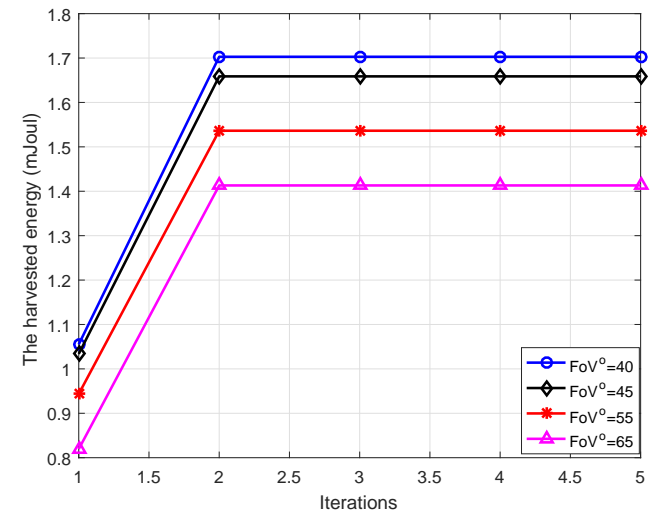

Fig. 2. The total harvested energy versus number of iterations, when $R_{t h}=$ 14 (Mbits/sec), $E_{t h}=1 \mu$ Joule, and the system model consists of 16 APs, 7 information users, and 4 EHUs with different receiver FoV.



Fig. 3. The total harvested energy versus the receiver FoV, when $R_{t h}=14$ (bits/sec), $E_{t h}=1 \mu \mathrm{Joule}$, and the system model consists of 16 APs with different number of IUs and EHUs.

the total harvested energy. All the simulation results are implemented under the simulation parameters given in Table I. Consider an $8 \times 8 \times 3 \mathrm{~m}^{3}$ room equipped with 16 VLC APs that are fixed in the ceiling, and serve several IUs and EHUs. Monte-Carlo simulations are used to assess the performance of the proposed algorithms, where every point in the numerical results is the average of implementing 100 different user realizations.

To illustrate the convergence behavior of Algorithm 1, Fig. 2 plots the total harvested energy at all EHUs versus the number of iterations, for three values of the FoV's. The figure shows the fast convergence of Algorithm 1 for all values of FoV's. The figure further shows that the total harvested energy increases as the FoV decreases. This is due to the fact that decreasing the $i$-th user's FoV strengthens the channels between the user and the APs that are in the user's field-ofview, which yields to a larger harvested energy.

Such result is further illustrated in Fig. 3, which plots the total harvested energy versus the FoV of users, for different number of IUs and EHUs, and by implementing both Algorithm 1 and the baseline approach. As expected, as the users' FoV increases, the total harvested energy decreases. Equation (4) further justifies this fact, since if the $F o V(\Theta)$ increases 
between $0^{\circ}$ and $90^{\circ}$, the channel decreases significantly. On the other hand, from Equation (4), decreasing the user's FoV decreases the probability of the coverage at that user. As a result, we can conclude that if the users' FoV is adjustable, decreasing its value subject to having at least one VLC AP in the FoV of that user would indeed increase the network harvested energy. Fig. 3 also shows that the proposed iterative Algorithm 1 outperforms the proposed baseline algorithm, with around $21 \%$ gain of harvested energy when the number of EHUs and IUs is 7 and 4, respectively, and 64\% when the number of EHUs and IUs is 4 and 7, respectively.

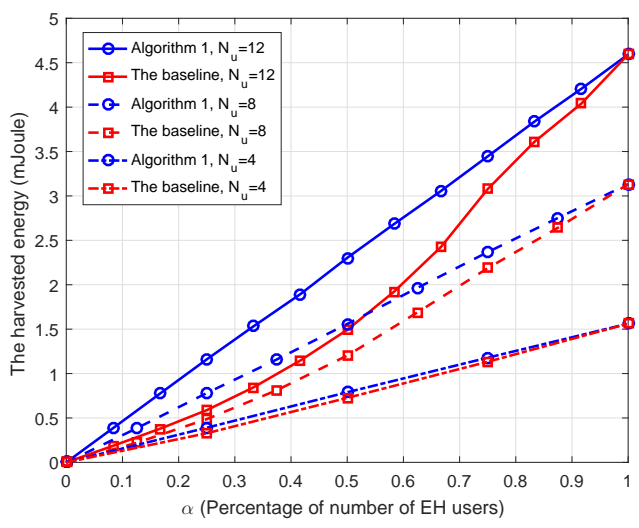

Fig. 4. The total harvested energy versus the precentage of number of EHUs out of the total number of users $(\alpha)$, when $R_{t h}=14(\mathrm{Mb} / \mathrm{sec}), E_{t h}=1$ $\mu \mathrm{Joule}$, and the system model consists of $16 \mathrm{APs}, \mathrm{FoV}=55^{\circ}$.

Fig. 4 plots the total harvested energy as a function of the fraction of $\alpha$, which denotes the percentage of number of EHUs out of the total number of users. As expected, as the fraction of EHUs increases, the total harvested energy increases. This is especially due to two main reasons. Firstly, for a fixed number of users $N_{u}$, as the fraction of EHUs increases, the number of EHUs increases, which adds to the overall objective function. Secondly, decreasing the number of IUs leads to decreasing the number of constraints in (15b), which increases the search space of (15b); thereby increasing the objective function. The figure further shows that, if $\alpha=$ 1 (i.e. when all users are EHUs), all the APs operate with a highest DC bias (i.e. $b_{i}=I_{H}, \quad i=1, . ., N_{A}$ ), and so both the iterative algorithm and the baseline achieve the same performance. On the other hand, if $\alpha=0$ (i.e., when all users are IUs), the total harvested energy becomes zero.

\section{CONCLUSION}

VLC-based systems are expected to play a major role in achieving the ambitious metrics of next generation wireless networks. This paper considers a VLC setup which considers the coexistence of both IUs and EHUs, and addresses the problem of maximizing the harvested energy by means of properly adjusting the DC bias values at the coordinating VLC APs subject to QoS constraints (minimum required data rate at IUs and minimum required harvested energy at EHUs). The paper solves such a difficult problem using an iterative algorithm by first approximating the problem to be a linear programming problem, and then by properly compensating for the approximation in an outer loop afterwards. Simulation results show that the proposed iterative algorithm converges rapidly for all values of the FoVs. The results further suggest that an appreciable harvested energy can be reached by optimizing the DC bias in VLC-based indoor communications.

\section{ACKNOWLEDGMENT}

This work was funded by the National Plan for Science, Technology and Innovation (Maarifah)-King Abdulaziz City for Science and Technology through the Science and Technology Unit at King Fahd University of Petroleum \& Minerals (KFUPM)-the Kingdom of Saudi Arabia, under grant number 15-ELE4157-04. The work was also supported by the Deanship of Scientific Research in KFUPM through grant number IN161023. Hayssam Dahrouj would like to thank Effat University in Jeddah, Saudi Arabia, for funding the research reported in this paper through the Research and Consultancy Institute.

\section{REFERENCES}

[1] P. H. Pathak, X. Feng, P. Hu, and P. Mohapatra, "Visible light communication, networking, and sensing: A survey, potential and challenges," IEEE commun. Surveys Tuts, vol. 17, no. 4, pp. $2047-$ 2077, 2015 .

[2] J. Miyakoshi, "Cellular and molecular responses to radio-frequency electromagnetic fields," Proc. IEEE, vol. 101, no. 6, pp. 1494-1502, 2013.

[3] H. Haas, L. Yin, Y. Wang, and C. Chen, "What is LiFi?" J. Lightw. Technol., vol. 34, no. 6, pp. 1533-1544, 2016.

[4] M. Obeed, A. M. Salhab, S. A. Zummo, and M.-S. Alouini, "Joint optimization of power allocation and load balancing for hybrid VLC/RF networks," IEEE/OSA J. Opt. Commun. and Netw., vol. 10, no. 5, pp. 553-562, 2018

[5] C. Li, W. Jia, Q. Tao, and M. Sun, "Solar cell phone charger performance in indoor environment," in IEEE Bioengineering Conf. (NEBEC), 2011, pp. 1-2.

[6] T. Rakia, H.-C. Yang, F. Gebali, and M.-S. Alouini, "Optimal design of dual-hop VLC/RF communication system with energy harvesting," IEEE Commun. Lett., vol. 20, no. 10, pp. 1979-1982, 2016.

[7] _ , "Dual-hop VLC/RF transmission system with energy harvesting relay under delay constraint," in Proc. IEEE Global Commun. Workshops (Globecom), 2016, pp. 1-6.

[8] Y. Li, N. Huang, J. Wang, Z. Yang, and W. Xu, "Sum rate maximization for VLC systems with simultaneous wireless information and power transfer," IEEE Photon. Technol. Lett., vol. 29, no. 6, pp. 531-534, 2017.

[9] A. M. Abdelhady, O. Amin, A. Chaaban, and M. S. Alouini, "Resource allocation for outdoor visible light communications with energy harvesting capabilities," in Proc. IEEE Global commun. Workshops (Globecom), Dec 2017, pp. 1-6.

[10] G. Pan, H. Lei, Z. Ding, and Q. Ni, "On 3-D hybrid VLC-RF systems with light energy harvesting and OMA scheme over RF links," in Proc. IEEE Global Commun. Conf. (Globecom), 2017, pp. 1-6.

[11] P. D. Diamantoulakis, G. K. Karagiannidis, and Z. Ding, "Simultaneous lightwave information and power transfer (SLIPT)," IEEE Trans. Green Commun. and Netw., 2018.

[12] R. Zhang, H. Claussen, H. Haas, and L. Hanzo, "Energy efficient visible light communications relying on amorphous cells," IEEE J. Sel. Areas Commun., vol. 34, no. 4, pp. 894-906, 2016.

[13] M. Obeed, A. M. Salhab, S. A. Zummo, and M.-S. Alouini, "Joint power allocation and cell formation for energy-efficient VLC networks," in Proc. IEEE Int. Conf. Commun. (ICC), Kansas, USA, 2018.

[14] J. M. Kahn and J. R. Barry, "Wireless infrared communications," Proc. IEEE, vol. 85, no. 2, pp. 265-298, 1997.

[15] J.-B. Wang, Q.-S. Hu, J. Wang, M. Chen, and J.-Y. Wang, "Tight bounds on channel capacity for dimmable visible light communications," J. Lightw. Technol., vol. 31, no. 23, pp. 3771-3779, 2013.

[16] M. Grant and S. Boyd, "Cvx: Matlab software for disciplined convex programming," 2008.

[17] S. Boyd and L. Vandenberghe, Convex Optimization. Cambridge University Press, 2004 